\title{
INVOLUÇÃO UTERINA, ATIVIDADE OVARIANA, PRIMEIRO CIO PÓS-PARTO E DISTÚRBIOS REPRODUTIVOS EM BÚFALAS
}

\author{
Sebastião Tavares Rolim Filho ${ }^{1}$, Haroldo Francisco Lobato Ribeiro ${ }^{2}$, William Gomes Vale ${ }^{2}$, Nayma \\ Da Silva Picanço ${ }^{1}$, Elizabeth Machado Barbosa ${ }^{3}$, Rafaela Nunes Ferreira ${ }^{1}$, André Reale Simões ${ }^{1}$
}

\author{
1 - Pós-graduando em Ciência Animal pela Universidade Federal do Pará \\ 2 - Professor Doutor da Universidade Federal Rural da Amazônia - UFRA - wmvale@hotmail.com \\ 3 - Mestre em Ciência Animal pela Universidade Federal do Pará
}

\section{RESUMO}

Objetivou-se com o presente estudo avaliar algumas características reprodutivas como a involução uterina, a atividade ovariana no pós-parto, o primeiro estro após o parto, e problemas reprodutivos em fêmeas bubalinas criadas no sistema misto (várzea e terra / pastagem artificial) em uma fazenda experimental do município de Belém , Pará, Brasil. Entre 2002 a 2005, um total de 167 períodos de pós-parto de 62 búfalas foram avaliados através de um exame clínico ginecológico e ultrassonografia. Os animais foram mantidos durante a estação chuvosa (janeiro-junho), em pastagens artificiais e, durante a estação seca (julho-dezembro), nas áreas de várzea, com excelente disponibilidade de pastagem. A retomada da atividade ovariana, verificada através da observação visual do primeiro estro do período do pósparto, ocorreu em média 47,06 $\pm 25,66$ dias, enquanto que a involução uterina verificada por palpação retal e exames ultrassonográficos foi de 27,5 \pm 7,77 dias. $\mathrm{O}$ número de casos de abortamentos, retenção da placenta e morte embrionárias foram de $10(5,90 \%), 13(7,78 \%)$ e $14(8,38 \%)$ casos, respectivamente, enquanto o número de casos de infecção uterina foi de 18 (10,7\%). A presença de ovários não-funcionais no decorrer do período do pós-parto foi observado em $26(15,56 \%)$ casos, tendo sido encontrados dois $(1,19 \%)$ casos de hipoplasia do ovário, três $(1,79 \%)$ casos de cistos ovarianos e dois $(1,19 \%)$ casos de salpingite.

PALAVRAS-CHAVE: Atividade ovariana, búfalos, cio pós-parto, distúrbios reprodutivos, eficiência reprodutiva.

\section{UTERINE INVOLUTION, OVARIAN ACTIVITY, FIRST POSTPARTUM ESTRUS AND REPRODUCTIVE DISORDERS IN BUFFALOES}

The aim of the present study was to evaluate some reproductive characteristics such as uterine involution, ovarian activity and first estrus after parturition, and reproductive disorders in buffaloes reared in the mixed system (floodplain and land / artificial pasture) in an experimental farm of Belém, Pará State, Brazil. A total of 62 female buffaloes and 167 postpartum periods were evaluated between 2002 to 2005 through gynecological and ultrasound examination. Animals were kept on artificial pasture during the rainy season (January-June) whereas on the floodplain areas with excellent availability of pasture during the dry season (July- 
December). The resumption of ovarian activity, verified by visual observation of the first estrus, was in average $47.06 \pm 25.66$ days, while the uterine involution verified by rectal palpation and ultrasound examination was 27.5 \pm 7.77 days. The number of cases of abortion, retained placenta and embryonic death were $10(5.90 \%), 13$
$(7.78 \%)$ and $14(8.38 \%)$ cases, respectively, while the number of cases of uterine infection was 18 (10.7\%). Regarding nonfunctional ovaries, $26(15.56 \%)$ cases were found and two $(1.19 \%)$ cases of ovarian hypoplasia, three $(1.79 \%)$ cases of ovarian cysts and two $(1.19 \%)$ cases of salpingitis were observed.

KEYWORDS: Buffaloes, postpartum ovarian activity, reproductive disturbances, reproductive efficiency.

\section{INTRODUÇÃO}

Segundo os dados mais recentes da Organização das Nações Unidas para a Agricultura e Alimentação (FAO, 2005), o Brasil apresentava um rebanho bubalino de 1.200 .700 cabeças em 2004. De acordo com o Ministério da Agricultura (BRASIL, 2005) e o Censo Agropecuário (IBGE, 2005) relativo ao ano de 2003, o efetivo do rebanho bubalino apresentava 1.149.000 cabeças, sendo que esses animais se distribuíam pelas cinco regiões do país nas seguintes quantidades/proporções: Norte 722.299 (62,9\%), Nordeste 106.117(9,2\%), Sudeste $104.449(9,1 \%)$, Sul $151.071(13,2 \%)$ e CentroOeste $64.872(5,6 \%)$.

Além do expressivo crescimento, pesquisas realizadas por diversos grupos demonstraram que os bubalinos apresentam grande produtividade $\mathrm{e}$ capacidade de adaptação às condições brasileiras (VALE, 1988; NOGUEIRA et al., 1989b; VALE et al., 1990; BARUSELLI et al., 1993). A exploração zootécnica de bubalinos caracteriza-se por apresentar boa eficiência reprodutiva e rápido desenvolvimento ponderal (VILLARES et al., 1979; VALE, 1988; NOGUEIRA et al., 1989;VALE et al, 1990). Portanto, as causas de baixa eficiência reprodutiva podem ser atribuídas a interações entre o genótipo e o meio ambiente incluindo-se o clima, manejo, nutrição e doenças.

Por outro lado, vários trabalhos em diferentes países (OBI REDDY et al., 1987;VALE, 1988; SHAH, 1990; VALE et al., 1990; BARUSELLI et al., 1993; ZICARELLI, 1994) têm enfatizado que a eficiência reprodutiva nos bubalinos é mais influenciada por fatores ambientais que genéticos. Dentre eles, o fotoperíodo, a precipitação pluviométrica, a umidade relativa do ar e a temperatura têm efeitos pronunciados sobre o comportamento reprodutivo da espécie. A importância de cada um desses fatores varia muito entre a situação geográfica, as condições de criação e o sistema de manejo.

O desempenho produtivo e reprodutivo da búfala está intimamente relacionado com a involução uterina e com a recuperação da atividade ovariana no pós-parto, por meio do surgimento de um novo ciclo estral, com crescimento de ondas foliculares, ovulação e fecundação (VALE \& RIBEIRO, 2005).

Estudos sobre involução uterina têm sido realizados em bovinos e reportados por vários autores. Dentre os principais fatores que influenciam a involução uterina são apontados o número de partos, fatores climáticos, hormonais, nutricionais, presença constante do bezerro ao pé da mãe e número de lactações (VALE, 1988; VALE et al., 1990; RIBEIRO, 1996). No Brasil, o estudo da involução uterina e da atividade ovariana no período pós-parto foi reportado pela primeira vez por VALE et al., (1986), os quais encontraram involução uterina e o primeiro ciclo estral pós-parto ocorrendo, em média, aos $28,6 \pm 6,6$ e $42,0 \pm 8,8$ dias, respectivamente.

Na região centro-sul do Brasil, em búfalas leiteiras da raça Murrah, BARUSELLI (1992) encontrou uma média de 36,6 dias pós-parto para o início da atividade ovariana, que foi marcada pela presença de um ciclo luteínico de curta duração $(9,9$ dias). Na região amazônica, RIBEIRO (1996), trabalhando com animais das raças Murrah e Mediterrânea criados em regime semi-extensivo, reportou variação na duração da involução completa do útero e cérvix. No mês de dezembro foi de $41,7 \pm 5,2$ dias, enquanto que nos meses de janeiro, fevereiro e março foi de $36,5 \pm 4,8,36,3 \pm 5,0$ e $34,4 \pm 3,2$ dias, respectivamente. O intervalo do parto ao primeiro estro observado foi de $124 \pm 30,06$ dias e esteve relacionado à precipitação pluviométrica e à oferta de pastagens. A involução uterina, medida pela avaliação dos diâmetros da cérvix e do corno uterino gestante e não gestante, em 580 observações, completou-se em média aos $37,2 \pm 4,5$ dias após o parto.

O desempenho reprodutivo da búfala depende intimamente da involução uterina, da continuidade 
da atividade ovariana no período pós-parto e do desencadeamento da ovulação e fertilização. De acordo com RIBEIRO (1996), a involução uterina na búfala ocorre com muita rapidez, sendo que o tamanho do útero diminui marcadamente nos primeiros 14 dias pós-parto, associado a uma concomitante descarga loquial rubra 24 a 96 horas após o parto e loquial alba entre o $16^{\circ}$ e $21^{\circ}$ dia do pós-parto.

Os ovários inativos e não funcionais são as causas mais importantes de anestro em fêmeas bubalinas. $\mathrm{O}$ anestro pode acontecer devido a causas nutricionais, relacionadas com proporção de energia, proteína, minerais e estresse térmico, produzindo um bloqueio do eixo hipotálamo-hipófise-ovário, com diminuição na circulação dos hormônios folículo estimulante (FSH), luteinizante ( $\mathrm{LH})$ e estrógeno, os quais contribuem para a inatividade ovariana (ZICARELLI, 1994). Portanto, quanto mais rápida ocorrer a involução uterina e o surgimento do primeiro cio no período do pós-parto, mais eficiente será a eficiência reprodutiva, significando maior desempenho econômico do rebanho.

Assim, objetivou-se com o presente estudo avaliar a eficiência reprodutiva através de características reprodutivas de bubalinos, criados na Unidade de Pesquisa Bubalina "Dr. Felisberto Camargo" - Embrapa Amazônia Oriental, por meio da investigação de registros de partos e problemas reprodutivos duarante o periodo de 2002 a 2005.

\section{MATERIAL E MÉTODOS}

Este trabalho foi realizado na Unidade de Pesquisa Bubalina "Dr. Felisberto Camargo" $\left(01^{\circ}\right.$ 26' 42.27" S e 48 $25^{\circ}$ '12.30" W), de propriedade do Centro de Pesquisa da Amazônia Trópico Úmido (CPATU-EMBRAPA), localizado em Belém, Pará, tipo climático tropical chuvoso, classificado como Ami, com estação chuvosa (janeiro a junho) e menos chuvosa (julho a dezembro), temperatura média anual de $26^{\circ} \mathrm{C}$, variando entre $22,6^{\circ} \mathrm{C}$ e $31,2^{\circ} \mathrm{C}$. A umidade relativa do ar é em média de $84 \%$, a precipitação pluviométrica anual é de $2.100 \mathrm{~mm}$ e a insolação anual total é de $2.091,5$ horas.

Os dados foram colhidos durante os anos de 2002 a 2005. Foram analisados um total 167 registros de partos e 62 fêmeas bubalinas das raças Murrah, Mediterrânea e Murrah x Mediterrânea.

Os animais foram manejados em regime de pastejo contínuo, na época menos chuvosa (julho a dezembro) e rotacionado intensivo na época mais chuvosa (janeiro a junho), em pastagens nativas de terra inundável em cuja composição botânica predominam gramíneas dos gêneros Paspalum e pastagens cultivadas de Brachiaria brizanta, Brachiaria humidicola e Panicum maximum (Tobiatã), nos quais permaneciam por aproximadamente três dias em cada piquete.

Os animais eram inseminados artificialmente com observação de cio e eram submetidos a exame de diagnóstico de gestação por ultra-som após 30 dias da inseminação, caso não ficassem gestantes eram acasaladas com o touro de repasse. Os animais gestantes eram reexaminados após 60 dias para confirmação da gestação e verificação da taxa de morte embrionária. Os animais com melhores escores de condição corporal $(3,0$ a 4,0$)$ eram inseminados em tempo fixo através do uso de hormonioterapia. $\mathrm{O}$ cio era observado diariamente por profissionais experientes, pela manhã, meio dia e ao final da tarde, além do uso de rufiões com busal marcador.

Todas as matrizes tiveram seus dados reprodutivos e produtivos documentados em fichas individuais, durante os exames ginecológicos, os quais eram realizados por médico veterinário qualificado duas vezes por semana, através de palpação retal seguindo metodologia preconizada por GRUNERT et al. (2005) e exame de ultra-som para a detecção de patologias reprodutivas, tais como endometrites, ovários afuncionais, hipoplasia ovariana, cistos foliculares e salpingites. Os dados de observações diárias eram anotados pelos ordenhadores ou inseminadores, tais como: data do cio, data do parto, abortamentos, retenção de placenta, coberturas, inseminações e touros utilizados.

\section{RESULTADOS E DISCUSSÃO}

O $1^{\circ}$ cio pós-parto, detectado pela manifestação clínica do cio, foi verificado neste estudo por meio da observação visual e do uso de rufiões com busal marcador e ocorreu em média aos $47,06 \pm 25,66$ dias. Assim sendo, o $1^{\circ}$ cio pós-parto, certamente está relacionado ao manejo reprodutivo ao qual foram submetidos os animais do presente experimento. Por sua vez, a involução uterina verificada pela palpação retal foi de $27,5 \pm 7,77$ dias, como mostrado na Tabela 1. 
Tabela 1 - Valores médios, desvio padrão e valores mínimo e máximo do primeiro cio pós-parto e involução uterina em búfalas criadas na Região Amazônica, através da observação visual do cio

\begin{tabular}{ccccc}
\hline Parâmetros & Média & DP & Min & Máx \\
\hline $1^{\circ}$ cio pós-parto & 47,06 dias & 25,66 & 19 dias & 120 dias \\
Involução uterina & 27,5 dias & 7,77 & 22 dias & 33 dias \\
\hline
\end{tabular}

EL-FADALY (1980 a; 1980b), verificando o efeito da cria ao pé e ordenha manual em búfalas egípcias leiteiras sobre a eficiência reprodutiva, encontrou intervalo médio pós-parto para o primeiro cio de 59,69 $\pm 25,40$ dias para as búfalas ordenhadas (22 a 120 dias) e, nas fêmeas com bezerro ao pé, o intervalo foi de 75,19 $\pm 24,37$ dias (38 a 135 dias).

Em revisão da espécie bubalina, SHAH et al. (1986) reportaram que a primeira ovulação pósparto ocorreu aos $96 \pm 22$ dias para o búfalo do pântano e aos 60-90 dias para o búfalo do rio, detectado pela análise de progesterona no plasma sanguíneo.

No Brasil, o estudo da involução uterina e da atividade ovariana no período do pós-parto foi relatado por VALE et al. (1986), os quais encontraram involução uterina e o primeiro ciclo estral pós-parto ocorrendo em média aos $28,6 \pm 6,6$ e 42,0 $\pm 8,8$ dias, respectivamente, semelhante aos dados encontrados no presente estudo. Os autores encontraram diferença entre o início da atividade ovariana no período pós-parto entre os grupos de búfalas submetidas ou não à separação dos bezerros à noite.

A literatura tem mostrado que as variações no tempo de anestro pós-parto dependem de inúmeros fatores (VALE, 1988; KHATTAB et al., 1990; SHAH et al., 1990). Contudo, em condições nutricionais e ambientais favoráveis, a atividade reprodutiva tende a se estabilizar por volta de 60 a 100 dias após o parto (VALE, 1988; SIMARAKS \& SUWANAKAMJAYA, 1991).

$\mathrm{Na}$ região centro-sul do Brasil, em búfalas leiteiras da raça Murrah, BARUSELLI (1992), encontrou em média 36,6 dias pós-parto para o início da atividade ovariana, que foi marcada pela presença de um ciclo luteínico de curta duração $(9,9$ dias). O primeiro estro seguido de comportamento foi observado em média aos 57,13 dias após o parto, com variação de 24 a 100 dias. O mesmo autor encontrou a atividade ovariana reiniciando-se a partir do $36^{\circ}$ dia do pós-parto. Segundo o autor, o primeiro cio comportamental ocorreu em média aos 57 dias, com variação de 24 a 100 dias.

Por outro lado, resultados superiores foram encontrados na Região Amazônica por RIBEIRO (1996) que, ao trabalhar com animais das raças Murrah e Mediterrânea, criados em regime semiextensivo, reportou o intervalo do parto ao primeiro estro aos $124 \pm 30,06$ dias relacionado à precipitação pluviométrica e à oferta de pastagens.

Em relação aos problemas reprodutivos como taxas de abortamento, retenção de placenta e morte embrionária, durante os anos de 2002 a 2005, acompanhando 62 vacas e 167 partos, foram observados $10(5,9 \%), 13(7,78 \%)$ e $14(8,38 \%)$ casos, respectivamente (Tabela 2).

Tabela 2 - Número de casos de abortamento, retenção de placenta, infecções uterinas e morte embrionária em um rebanho bubalino, na Região Amazônica, em um total de 62 vacas, durante o período de 2002 a 2005

\begin{tabular}{lcc}
\hline Tipo de alteração & $\mathrm{N}^{\circ}$ de casos & Percentual (\%) \\
\hline Abortamento & 10 & 5,90 \\
Retenção de placenta & 13 & 7,78 \\
Infeç̧ões uterinas & 18 & 10,77 \\
Morte embrionária & 14 & 8,38 \\
Ovários afuncionais & 26 & 15,56 \\
Hipoplasia ovariana & 2 & 1,19 \\
Cistos foliculares & 3 & 1,79 \\
Salpingites & 2 & 1,19 \\
Total & 88 & 52,56 \\
\hline
\end{tabular}

O abortamento em bubalinos tem sido associado a doenças como neosporose, brucelose, leptospirose e rinotraqueíte infecciosa bovina (IBR), as quais acometem os búfalos de modo semelhante aos bovinos (LAU, 1990; FAINE et al., 1999), estando presentes em níveis elevados em búfalos da região estudada, mas não há estimativas de taxas nem notícia de surtos epidêmicos de abortamentos ocasionados pelas enfermidades acima mencionadas.

VALE et al. (1994) observaram que, em um rebanho com 63 animais submetidos à inseminação artificial, 2,4\% dos animais apresentaram retenção de placenta e 7,1\% apresentaram morte embrionária. BARUSELLI et al. (1997) registraram uma taxa de $7 \%$ de morte embrionária, sem causa definida.

$\mathrm{O}$ número de casos de infecção uterina, em um total de 167 partos, foi de 18 (10,77\%). No 
Brasil, são poucas as citações de ocorrência de endometrites na búfala. RIBEIRO et al. (1987), em estudos na mesma região encontraram $17(2,70 \%)$ casos de endometrite crônica suave. VALE et al. (1994) observaram que em um rebanho com 63 animais submetidos à inseminação artificial, 7,2\% apresentavam infecção uterina.

Em relação aos ovários afuncionais, foram encontrados 26 casos ou 15,56 \%, analisando um total de 62 animais, em diferentes épocas do ano. Segundo DWIVEDI \& SHIGH (1971), o ovário afuncional é a alteração de maior frequência e importância apresentada pelo ovário da búfala. Entretanto, as taxas encontradas neste trabalho foram inferiores aos $36,7 \%$ reportados por DESSOUKI \& JUMA (1973); incidência inferior à reportada por CHAUDHRY et al. (1978), porém superior às encontradas por ELWISHY et al. (1971).

RIBEIRO et al. (1987) registraram que os ovários afuncionais são alterações causadas principalmente por erros de manejo, deficiências nutricionais e doenças caquetizantes, sendo a alteração mais frequente no ovário da búfala, com $102(16,21 \%)$ casos, semelhante aos dados reportados neste estudo.

Foram observados 2 (1,19\%) casos de hipoplasia ovariana em um total de 62 animais examinados, taxas superiores às citadas na literatura que estabelece variação na incidência de hipoplasia ovariana entre $0,33 \%$ a $0,97 \%$ (SHALASH, 1958; RAO \& KESAVAMURTHY, 1971).

Na búfala, a incidência de cisto folicular varia de $0,19 \%$ a $5,9 \%$ (SINGH et al., 1979), média semelhante aos dados apresentados neste estudo. De acordo com RAO \& KESAVAMURTHY (1971), a ocorrência de cisto folicular é baixa em comparação com vacas bovinas leiteiras. Nessa espécie, os cistos podem estar associados à ninfomania (ROBERTS, 1986), o que não foi observado em búfalas por RAO \& RAJYA (1976).

Foram observados 2 (1,19\%) casos de salpingites neste estudo. De acordo com a literatura, as salpingites são frequentemente secundárias a processos inflamatórios no útero. As hidrossalpingites, por sua vez, têm sido observadas não só em consequência da aplasia segmentar como também devido à aderência das extremidades distal ou proximal, ou ao longo de todo o oviduto (SHOKEIR, 1958; SHARMA et al., 1967; DWIVEDI \& SINGH, 1971; RAO \& RAJYA, 1976;
VALE et al., 1988).

RIBEIRO et al. (1987) observaram 27 $(4,29 \%)$ casos de alterações de oviduto, incidência superior à encontrada na presente pesquisa; porém, somente $2(0,31 \%)$ casos de salpingites. As alterações de oviduto em búfalas ocupam lugar de destaque como causa da baixa eficiência reprodutiva nesta espécie (BHATTACHARYA, 1974). Esses autores indicam a existência de uma relação entre o nível de inflamação uterina e a aparição de alterações de oviduto.

Com base na palpação retal, a primeira ovulação foi observada aos $38 \pm 2$ e $69 \pm 21$ dias em búfalas leiteiras no Egito e na Índia, respectivamente (EL-FADALY, 1980 a; 1980b; CHAUHAN et al., 1977). EL-FOULY et al. (1976) encontraram diferenças significativas em búfalas com ou sem bezerro ao pé no reinício da atividade ovariana e no primeiro cio pós-parto.

\section{CONCLUSÃO}

O primeiro cio pós parto está diretamente relacionado ao tipo de manejo ao qual estão submetidos os animais, apresentando-se, neste estudo, em média aos 47,06 dias, já a involução uterina apresentou-se aos 27,5 dias dentro do intervalo apresentado na literatura. Assim, pelo tipo de manejo utilizado, foi possível proporcionar uma melhoria na eficiência reprodutiva do rebanho estudado. Desta forma, pode-se inferir que o tipo de manejo reprodutivo proporcionado foi sem dúvida um fator fundamental para a diminuição do período de intervalo entre partos e do primeiro cio.

\section{REFERÊNCIAS}

BARUSELLI B.P. Manejo reprodutivo em bubalinos. 1993. 46p. Monografia (....) -Instituto de Zootecnia, Nova Odessa, SP, 1993.

BARUSELLI, P. S.; MUCCIOLO, R. G.; VISINTIN, J. A.; VIANA, W. G.; ARRUDA, R. P.; MADUREIRA, E. H.; OLIVEIRA, C. A.; MOLERO-FILHO, J. R. Ovarian follicular dynamics during the estrous cycle in buffalo (Bubalus bubalis). Theriogenology, v. 47, n. 8, p. 15311547, 1997.

BARUSELLI, P.S. Atividade ovariana e comportamento reprodutivo no período pós-parto em búfalos (Bubalus bubalis). São Paulo, 1992, 99p. Dissertação (Mestrado) - Faculdade de Medicina Veterinária e Zootecnia, Universidade de São Paulo. http://www.fmvz.usp.br/index.php/site/pos_graduacao/bi- 
blioteca_digital_de_teses_e_dissertacoes

BHATTACHARYA, P. Reproduction. In: COCKRIL, W. $\mathrm{R}$. The husbandry and health of the water buffalo. 1 ed. Rome: FAO, 1974. 993 p.

BRASIL. Ministério da Agricultura. Rebanho bubalino brasileiro - Efetivo por Estado. 1983. Disponível em :< www.agricultura.gov.br>. Acesso em: 02 out 2005.

CHAUDHRY, R. A.; SAMAD, H. A.; AHMED, W. Clinical incidence of reproductive disorders in buffalo at Faisalabad. FAO/SIDA Felow-up Seminar on Animal Reproduction, Faisalabad, Pakistan, p.1-5. 1978.

CHAUHAN, F.S.; SINGH, N.; SINGH, M. Involution of the uterus and cervix in bufaloes. Indian Journal Dairy Science, v. 30, p.286-289. 1977.

DESSOUKY, F.; JUMA, K. H. Infertility problems among cows buffaloes in Iraq. Indian Journal Animal Science. New Delhi, v. 43, n. 3, p. 187-92, 1973.

DWIVEDI, J. N.; SINGH, C. M. Studies on the pathology of female reproductive organs of Indian buffalo. IV. Observations on bursitis and hydrosalpinx cases. Indian Journal of Animal Science, v. 41, p. 27-32, 1971.

EL-FADALY, M.A. Effect of suckling and milking on the breeding efficiency of buffaloes. I. First postpartum oestrus. Veterinary Medical Journal, v.28, p.399-344. 1980a.

EL-FADALY, M.A. Effect of suckling and milking on the breeding efficiency of buffaloes. II. Uterine Involution . Veterinary Medical Journal, v.28, p.353-359, 1980b.

EL-FOULY, M. A.; KOTBY, E. A.; EL-SOBHY, H. E. Effect of suckling on uterine and cervical involution in post-partum Egyptiam buffaloes. Indian Journal Animal Science, v. 46, p.221-227, 1976.

ELWISHY, A. B.; ELSAWAF, S. A.; ELMIKKAWI, F.; OMAR, A. A. Monthly and seasonal variation in sexual activity of male Damascus goats. Indian Journal of Animal Sciences, New Delhi, v. 41, n. 7, p. 562-569, 1971.

F.A.O. FAOSTAT. Data Base 2004. Disponível em: $<$ http://faostat.fao.org/faostat/collections?Version $=$ ext\&hasbulk $=0>$. Acesso em: 14 ago 2005 .

FAINE, S.; ADLER, B.; BOLIN, C. et al. Leptospira and Leptospirosis. 2. ed. Melbourne: MediSci, 1999. 272 p.

LAU, H. D. Common buffalo diseases in Amazonian Brazil. Buffalo Bulletin, v. 9, p. 75 -77, 1990.

McENTEE, K. Reproductive pathology of domestic mammals. 1 ed. Academic Press, Inc. 1990. 401 p.

NOGUEIRA, J.R.; BARBOSA, C.; MATTOS, J.C.A.; CAMPOS, B.E.S.; CAMARGO, D.F.V. Peso ao nascer e desenvolvimento ponderal de bubalinos da raça Mediter- râneo e Jafarabadi. Boletim de Indústria Animal, v. 46, p. 193-198, 1989a.

NOGUEIRA, J.R.; BARBOSA, C.; MATTOS, J.C.A; CAMPOS, B.E.S.; CAPELLOZA, C.N.Z. Aspectos da eficiência reprodutiva de bualinos das raças Mediterâneo e Jafarabadi. Boletim de Indústria Animal, v. 46, p. 45$54,1989 b$.

OBI-REDDY, A.; TRIPATHI, V.N.; RAINA, V.S. Effect of climate on the incidence of oestrus and conception rate in murrah buffaloes. Indian Journal of Animal Science, v. 57, p. 204-207, 1987.

RAO, A.V.N; KESAVAMURTHY, A. Study on reproductive disorder in buffalo cows of Andhra Pradesh. I. Incidence of anatomical and physiologically causes. Indian Veterinary Journal. Madras. v. 48, p. 1007-1014. 1971.

RAO, P. R.; RAJYA, B. S. A. Phatoanatomy of the female genital tract of buffaloes. Indian Journal Animal Science New Delhi, v. 46, n. 3, p. 125-130, 1976.

RIBEIRO, H. F. L.; SILVA, N. Q.; VALE, W. G. Distúrbios reprodutivos de búfalas na região do baixo amazonas, Brasil. Pesquisa Agropecuária Brasileira Rio de Janeiro, v. 7 , n. 4, p. 113-115, 1987 ..

ROBERTS, S.J. Veterinary obstetrics in genital diseases. Theriogenology. 3 ed. Michigan: Edwards Brothers, 1986. 955 p.

SHAH, S.N.H. Prolonged calving intervals in Nili-Ravi buffalo. Tese (Doutorado) - Utrecht: Rijksuniversiteit te Utrecht, 162p., 1990.

SHALASH, M. R. Physiology of reproduction in the buffalo cow. International Journal of Fertility, v. 3, p. 425432, 1958.

SHARMA, O. P.; BHALLA, R. C.; SONI, B. K. Study on some aspects of ovarian abnormalities as a cause of infertility on buffalo - cows. Indian Veterinary Journal, v. 44, p. 504-508. 1967.

SHOKEIR, A. A. Observation on discases conditions and malformation of reproductive organs of slaughtered buffaloes. Veterinary Medicine Journal Giza, v. 5, p. 256286, 1958.

SINGH, N.; CHAUHAN, F. S.; SINGH, M. Postpartum ovarian activity and fertility in buffaloes Indian. Journal Dairy Science, v. 32, n. 2, p. 134-139, 1979.

VALE WG, RIBEIRO HFL, SOUSA JS, OHASHI OM. Involução uterina e atividade ovariana pós-parto em búfalas. Revista Brasileira de Reprodução Animal, v.10, p.187-192, 1986.

VALE, W. G.; OHASHI, O. M.; SOUSA, J. S.; RIBEIRO, H. F. L. Studies on the reproduction of water buffalo in the Amazon basin. In: Livestock Reproduction in Latin America. Joint FAO/IAEA division of Nuclear Techniques in food and Agriculture, Vienna, p.201-210, 1990. 
VALE, W. G.; SOUSA, J. S.; RIBEIRO, H. F. L.; OHASHI, O. M.; LÁU, H. D.; SILVA, A. O. A. Preparation of Teaser " buffalo bull goes uses in artificial programs. Buffalo Journal, v. 10, n.1, p. 75-79, 1994.

VALE, W.G. Bubalinos: fisiologia e patologia da reprodução. Campinas, Fundação Cargill, 86p., 1988.

VALE, W.G; RIBEIRO, H.F.L. Características reprodutivas dos bubalinos: puberdade, ciclo estral, involução uterina e atividade ovariana no pós-parto. Revista Brasileira de Reprodução Animal, Belo Horizonte, v.29, n.2, p.6373, 2005.

VILLARES, J. B.; RAMOS, A. A.; ROCHA, G. P. Eficiência reprodutiva de búfalos Jafarabadi nos Vales do Tietê e Ribeira, em São Paulo, Brasil. Bubalinos, Campinas, v. 1, p. 253-276, 1979.

ZICARELLI, L. Management in different environmental condition. Buffalo Journal, v. 44, Suplemento 2, p.1738, 1994.

Protocolado em 05 mar. 2010. Aceito em: 17 fev. 2011. 